\title{
Enhancement via Fusion of Mammographic Features
}

\author{
Iztok Koren $^{\dagger}$, Andrew Laine ${ }^{\ddagger}$, and Fred Taylor ${ }^{\dagger}$ \\ ${ }^{\dagger}$ Dept. of Electrical and Computer Engineering \\ ${ }^{\ddagger}$ Center for Biomedical Engineering \\ University of Florida, Gainesville, FL 32611 \\ Columbia University, New York, NY 10027
}

\begin{abstract}
Mammographic image enhancement methods are typically aimed at either improvement of the overall visibility of features or enhancement of a specific sign of malignancy. In this paper, we present a synthesis of the two paradigms by means of image fusion. After a redundant B-spline wavelet transform decomposition is carried out, the transform coefficients are processed for enhancement of microcalcifications, circumscribed masses, and stellate lesions. The modified coefficients are then fused for reconstruction of an enhanced image with improved visualization of malignancies. Both processing for enhancement of selected features and fusion of the resultant images are accomplished within a single wavelet transform framework which contributes to the computational efficiency of the described method. The devised algorithm not only allows for efficient combination of specific features of importance in the contrast enhanced images, but also provides a flexible framework for incorporation of different enhancement methods and their independent optimization.
\end{abstract}

\section{Introduction}

Mammography is the best method for early detection of breast cancer at a time when approximately 80 percent of women diagnosed with breast cancer have no identifiable risk factors for this disease. The early detection of breast cancer is essential since therapeutic actions are more likely to be successful in the early stages. Finding small malignancies and subtle lesions is often difficult with false-negative rate being due both to difficulty with discerning subtle features on the complex normal anatomy background and oversight of abnormalities.

Contrast enhancement can make more obvious unseen or barely seen features of a mammogram without requiring additional radiation. Better visibility of suspicious structures can increase effectiveness and efficiency, and thus improve the diagnostic performance of mammography.

Existing methods of mammographic image enhancement can be divided roughly into two categories: (1) methods aimed at better visualization of all features present in an image $[1,2,10,12]$, and (2) methods that target specific features of importance (e.g., microcalcifications [13, 14, 16], stellate lesions [8]).

Methods from the first category are not optimized for a specific type of cancer and sometimes not even for mammography. Rather, they try to improve the perceptual quality of the entire image and are often developed with a framework more general than mammography alone in mind.

The second category methods concentrate on revelation of particular signs of malignancy. They can be very successful in their area of specialization; however, in order to process mammogram for presence of various features, one would need to apply different algorithms independently resulting in both larger number of images to be interpreted by a radiologist and increased computational complexity of such a procedure.

In this paper, we present an approach which overcomes these shortcomings and problematic limitations via synthesis of the two paradigms by means of image fusion.

\section{Methodology}

The goal of our method is to adapt specific enhancement schemes for distinct mammographic features, and then combine the set of processed images into an enhanced image. The mammographic image is first processed for enhancement of microcalcifications, masses, and stellate lesions. From the resulting enhanced images, the final enhanced image is synthesized by means of image fusion [7]. Wavelet based image enhancement and fusion are merged into a unified framework, so that there is no need for carrying out the two operations independently (i.e., computing wavelet decompositions, modifying wavelet coefficients for enhancement of specific features, reconstructing the enhanced images, performing wavelet transforms of the enhanced images, fusing transform coefficients, and obtaining the final result by reconstruction from fused wavelet coefficients). Both enhancement and fusion are therefore implicit (i.e., performed in the wavelet domain only). 


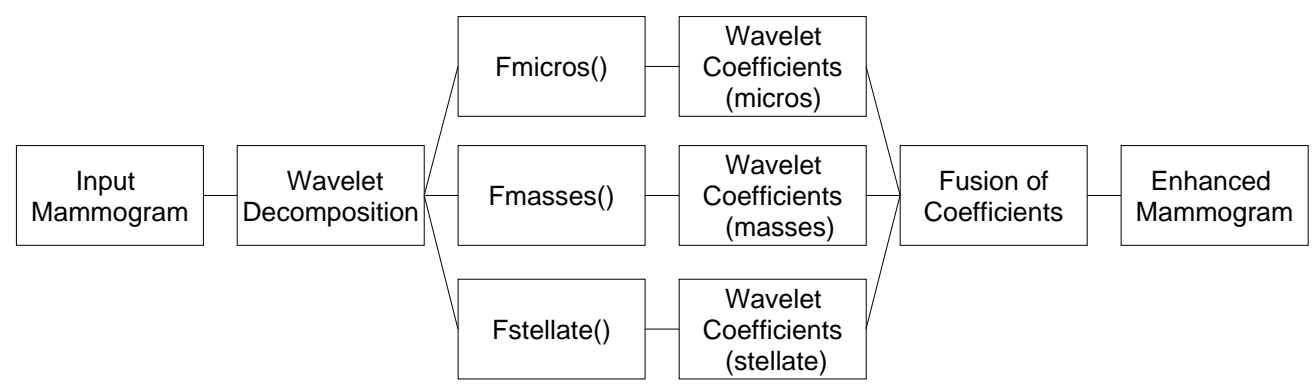

Figure 1: Overview of the algorithm.

Figure 1 presents a block scheme of the overall algorithm.

The algorithm consists of two major steps: (1) wavelet coefficients are modified distinctly for each type of malignancy; (2) the obtained multiple sets of wavelet coefficients are fused into a single set from which the reconstruction is computed. The devised scheme allows efficient deployment of an enhancement strategy appropriate for clinical screening protocols: enhancement algorithm is first developed for each specific type of feature independently, and the results are then combined using an appropriate fusion strategy.

The structure of the algorithm also enables independent development and optimization of enhancement strategies for individual mammographic features as well as the fusion module.

\subsection{B-Spline Wavelet Transform}

Since diagnostic features in mammograms appear in a variety of shapes and sizes, traditional image enhancement techniques such as histogram equalization and unsharp masking seldom produce satisfactory results and are clearly outperformed by more sophisticated methods $[10,12]$. Recognizing the benefit of mammographic image processing across different scales have resulted in a variety of wavelet-based techniques; however, the choice of an appropriate wavelet transform is of crucial importance. In enhancement of mammograms, for example, it is essential to improve the visibility of features without distorting their appearance and shape. Algorithm introduced artifacts are dangerous since they can lead to misdiagnosis, and lack of translation invariance of the transform has been identified as a possible source of artifacts. In the light of this major shortcoming of orthogonal and biorthogonal wavelet transforms, translation-invariant overcomplete wavelet representations of signals have become popular $[2,10,13,14]$.

In two dimensions, the lack of rotation invariance affects the processing results as well, and several steerable [3] wavelet transforms have been devised to ad- dress this problem $[1,8,9]$. Translation and rotation invariance are equally important for image fusion applications, and our experiments have shown elimination of orthogonal and biorthogonal wavelet transform caused artifacts when the steerable dyadic wavelet transform based fusion method [7] has been used.

Here, we employ a generalization of the discrete dyadic wavelet transform [11] with wavelets being equal to the second derivative of a central B-spline. This multiscale spline derivative-based transform [5] has several nice properties: (1) it is translationinvariant and approximately steerable, (2) it is well suited for incorporating flexibility from a variety of methods $[1,2,4,8,10,13,14]$, and (3) it can be implemented as a filter bank consisting of one-dimensional filters only.

The wavelets can be expressed as

$$
\psi(x, y)=\frac{\partial^{2} \varrho(x, y)}{\partial x^{2}},
$$

where

$$
\varrho(x, y)=\beta_{p+2}(x) \beta_{p+2}(y)
$$

and $\beta_{p}(x)$ denotes a central B-spline of order $p \in \boldsymbol{N}$.

Since central B-splines can closely approximate a Gaussian probability density function (in fact, they converge to a Gaussian as their order tends to infinity), $\varrho(x, y)$ can be made approximately circularly symmetric and, consequently, $\psi(x, y)$ approximately steerable.

A rotation of $\psi(x, y)$ by $\theta_{0}$ can therefore be approximated by

$$
\begin{aligned}
\psi^{\theta_{0}}(x, y) \simeq\left(\left(\cos \theta_{0}\right)^{2} \frac{\partial^{2}}{\partial x^{2}}+\right. & 2 \cos \theta_{0} \sin \theta_{0} \frac{\partial^{2}}{\partial x \partial y}+ \\
& \left.+\left(\sin \theta_{0}\right)^{2} \frac{\partial^{2}}{\partial y^{2}}\right) \varrho(x, y),
\end{aligned}
$$

so that the set of basis functions that approximately steer the wavelets $\psi(x, y)$ is $\left\{\frac{\partial^{2} \varrho(x, y)}{\partial x^{2}}, \frac{\partial^{2} \varrho(x, y)}{\partial x \partial y}\right.$, $\left.\frac{\partial^{2} \varrho(x, y)}{\partial y^{2}}\right\}$.

Figure 2 shows the building blocks of a filter bank implementation of the transform which uses the set of 


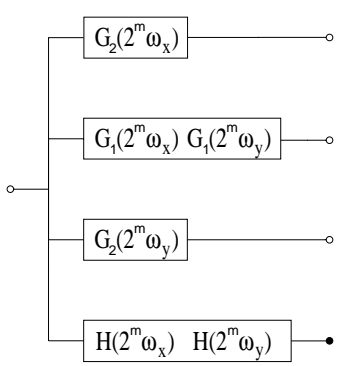

(a)

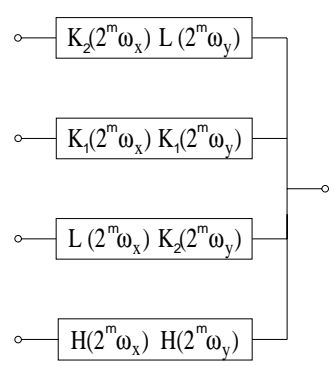

(b)
Figure 2: Filter bank implementation of a multiscale spline derivative-based transform with a second derivative wavelet. Basic processing modules for (a) decomposition and (b) reconstruction at a scale $2^{m}$.

basis functions needed to approximately steer $\psi(x, y)$. The filters were specified as

$$
\begin{gathered}
G_{d}(\omega)=e^{j \omega\left(\frac{2-d}{2}\right)}\left(2 j \sin \left(\frac{\omega}{2}\right)\right)^{d}, \\
H(\omega)=\left(\cos \left(\frac{\omega}{2}\right)\right)^{2 p}, \quad L(\omega)=|H(\omega)|^{2}, \quad \text { and } \\
K_{d}(\omega)=\frac{1}{(2 j)^{d}}\left(e^{-j \omega\left(\frac{2-d}{2}\right)} \sin \left(\frac{\omega}{2}\right)\right)^{2-d} \cdot \\
\cdot \sum_{m=0}^{2 p-1}\left(\cos \left(\frac{\omega}{2}\right)\right)^{2 m},
\end{gathered}
$$

where $d \in\{1,2\}$.

All the filters are either symmetric or antisymmetric, a property which enables an additional speedup when a symmetric (e.g., mirror extended) input signal is used [6].

B-spline approximations are also well suited for carrying out the proper initialization of the wavelet transform by means of simple prefiltering. Such a spline based initialization can significantly improve the accuracy of processing at finer scales.

\subsection{Enhancement and Fusion}

After the wavelet decomposition, the obtained coefficients are modified for improved visualization of features of diagnostic importance. Local enhancement of microcalcifications, circumscribed masses, and stellate lesions has been developed for each type of malignancy separately. An advantage of the method represented by Figure 1 is the fact that the entire processing algorithm can be split into subproblems which can be tackled independently.

For enhancement of microcalcifications, second derivatives along directions of $x$ and $y$-axis are added to form an approximation to a Laplacian of Gaussian. The obtained coefficients are thresholded, and the original coefficients at the corresponding locations multiplied by a gain factor. Similar enhancement through detection was used by Strickland and Hahn $[13,14]$. To reduce the number of false-positive elongated samples, the strength of local orientation was computed by employing second derivative wavelets in conjunction with their Hilbert transform pairs for multiscale orientation analysis. Note that, although not implemented for the purpose of this paper, it is possible to obtain voices of the transform as well (e.g., octaves "2.5" and "3.5" $[13,14])$; central B-spline properties enable computation of the transform at any integer scale [15].

Enhancement of circumscribed masses is carried out by applying a piecewise linear enhancement function [2] to wavelet coefficients at scales $2^{3}$ through $2^{5}$. The selection of the scale range was based upon the pixel resolution $(116 \mu \mathrm{m})$ of digitized mammograms in the University of Florida database.

Stellate lesions are contrast enhanced according to the observation that they introduce a distortion into a radial orientation pattern from the nipple to the chest wall [4]. The 1-norm of differences between local orientation and average orientation within a sliding window is used as an input to a soft thresholding function at each dyadic scale independently [8].

The choice of enhancement parameters controls the aggressiveness/subtleness of each resultant enhancement (i.e., prominence of the targeted feature with respect to the surrounding tissue).

Note also that it is possible to put different weights on features, and exclude certain features from the final result.

\section{Experimental Results}

Our method was applied to digitized mammograms from the University of Florida database, and showed promising results in terms of improved visibility and detection of subtle features.

Figure 3 demonstrates the results of processing using the multiscale analysis contrast enhancement algorithm [10], and using the proposed fusion of enhanced features method. In this example, the fusion of enhanced features emphasizes the appearance of a mass which is surrounded by dense parenchyma of the breast. Our preliminary results suggest that this type of image is more easily interpreted by radiologists compared to images produced via global enhancement techniques; however, the multiscale analysis contrast enhancement algorithm [10] is being refined as well. A powerful aspect of the enhancement via fusion scheme lies in its flexibility: the multiscale analysis based global contrast enhancement algorithm [10] can be readily incorporated into the scheme as one of the branches before the fusion module. 


\section{Conclusion}

The described method incorporates a variety of properties of mammographic image enhancement methods tailored to specific signs of malignancy into a unified computational framework. Multiscale spline derivative-based transform has proved flexible enough for implicit enhancement of individual types of mammographic features and thus enabled processing within a single wavelet transform decomposition. In addition to its efficiency, the algorithm is also well suited for further refinements; optimizations can be performed for each type of malignancy alone, and separately for the fusion strategy.

Our preliminary experiments imply that the enhancement via fusion approach can provide more obvious clues for radiologists. Further clinical tests are planned to verify that the versatility of this paradigm can provide a better viewing environment for an easier and a more reliable interpretation of mammograms.

\section{Acknowledgment}

This work was supported by the U.S. Army Medical Research and Materiel Command under DAMD17-96$1-6093$.

\section{References}

[1] C.-M. Chang and A. Laine, "Enhancement of mammograms from oriented information," in Proc. IEEE Int. Conf. Image Process., Santa Barbara, CA, Oct. 1997, vol. 3, pp. 524-527.

[2] J. Fan and A. Laine, "Multiscale contrast enhancement and denoising in digital radiographs," in Wavelets in Medicine and Biology, A. Aldroubi and M. Unser, Eds., CRC Press, Boca Raton, FL, 1996, pp. 163-189.

[3] W. T. Freeman and E. H. Adelson, "The design and use of steerable filters," IEEE Trans. Pattern Anal. Machine Intell., vol. 13, pp. 891-906, 1991.

[4] W. P. Kegelmeyer, J. M. Pruneda, P. D. Bourland, A. Hillis, M. V. Riggs, and M. L. Nipper, "Computer-aided mammographic screening for spiculated lesions," Radiology, vol. 191, pp. 331-337, 1994.

[5] I. Koren, A Multiscale Spline Derivative-Based Transform for Image Fusion and Enhancement, Ph.D. thesis, Department of Electrical and Computer Engineering, University of Florida, Gainesville, FL, 1996.

[6] I. Koren and A. Laine, "A discrete dyadic wavelet transform for multidimensional feature analysis," in M. Akay (Editor), Time-Frequency and
Wavelets in Biomedical Signal Engineering, New York, NY: IEEE Press, 1998, pp. 425-449.

[7] I. Koren, A. Laine, and F. Taylor, "Image fusion using steerable dyadic wavelet transform," in Proc. IEEE Int. Conf. Image Process., Washington, D.C., Oct. 1995, vol. 3, pp. 232-235.

[8] I. Koren, A. Laine, F. Taylor, and M. Lewis, "Interactive wavelet processing and techniques applied to digital mammography," in Proc. IEEE Int. Conf. Acoust. Speech Signal Process., Atlanta, GA, May 1996, vol. 3, pp. 1415-1418.

[9] A. Laine, I. Koren, W. Yang, and F. Taylor, "A steerable dyadic wavelet transform and interval wavelets for enhancement of digital mammography," in Wavelet Applications II, H. H. Szu, Ed., Proc. SPIE, Orlando, FL, Apr. 1995, vol. 2491, pp. $736-749$.

[10] A. F. Laine, S. Schuler, J. Fan, and W. Huda, "Mammographic feature enhancement by multiscale analysis," IEEE Trans. Med. Imaging, vol. 13, pp. 725-740, 1994.

[11] S. Mallat and S. Zhong, "Characterization of signals from multiscale edges," IEEE Trans. Pattern Anal. Machine Intell., vol. 14, pp. 710-732, 1992.

[12] W. M. Morrow, R. B. Paranjape, R. M. Rangayyan, and J. E. L. Desautels, "Region-based contrast enhancement of mammograms," IEEE Trans. Med. Imaging, vol. 11, pp. 392-406, 1992.

[13] R. N. Strickland and H. I. Hahn, "Wavelet transform matched filters for the detection and classification of microcalcifications in mammography," in Proc. IEEE Int. Conf. Image Process., Washington, D.C., Oct. 1995, vol. 1, pp. 422-425.

[14] R. N. Strickland and H. I. Hahn, "Wavelet transforms for detecting microcalcifications in mammograms," IEEE Trans. Med. Imaging, vol. 15, pp. 218-229, 1996.

[15] M. Unser, A. Aldroubi, and S. J. Schiff, "Fast implementation of the continuous wavelet transform with integer scales," IEEE Trans. Signal Process., vol. 42, pp. 3519-3523, 1994.

[16] H. Yoshida, W. Zhang, W. Cai, K. Doi, R. M. Nishikawa, and M. L. Giger, "Optimizing wavelet transform based on supervised learning for detection of microcalcifications in digital mammograms," in Proc. IEEE Int. Conf. Image Process., Washington, D.C., Oct. 1995, vol. 3, pp. 152-155. 


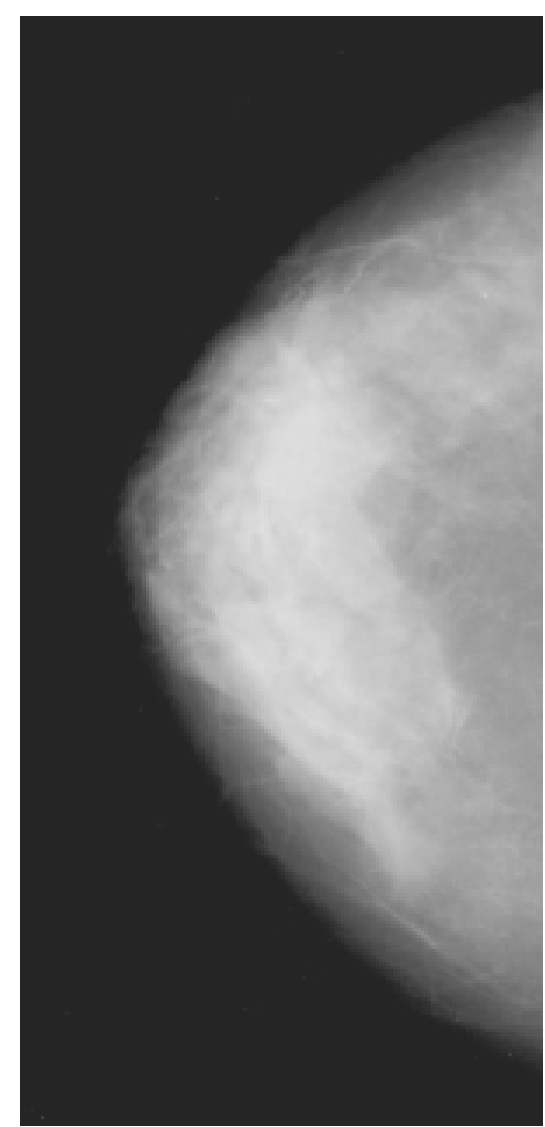

(a)

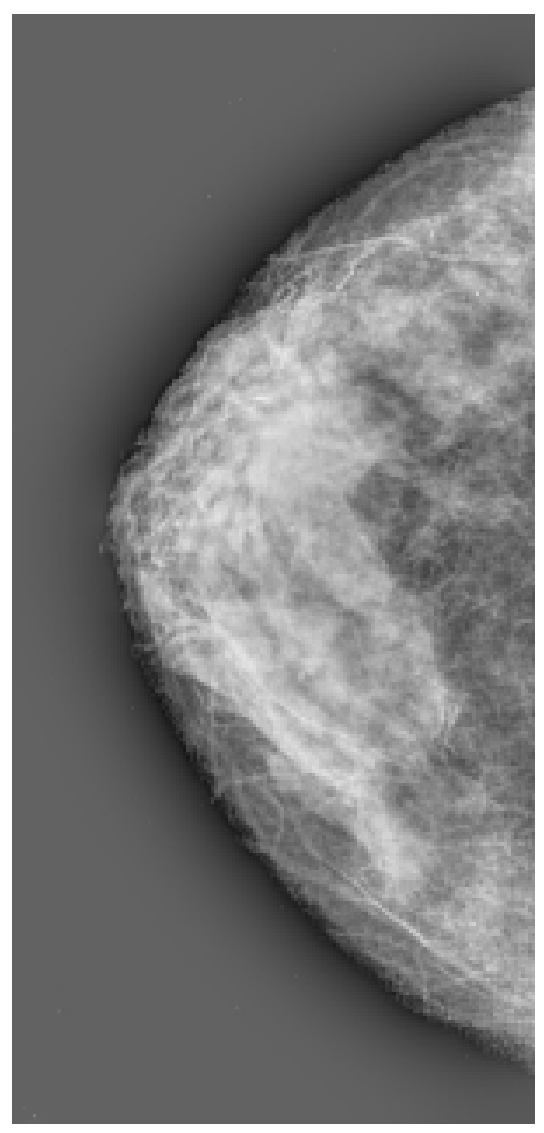

(b)

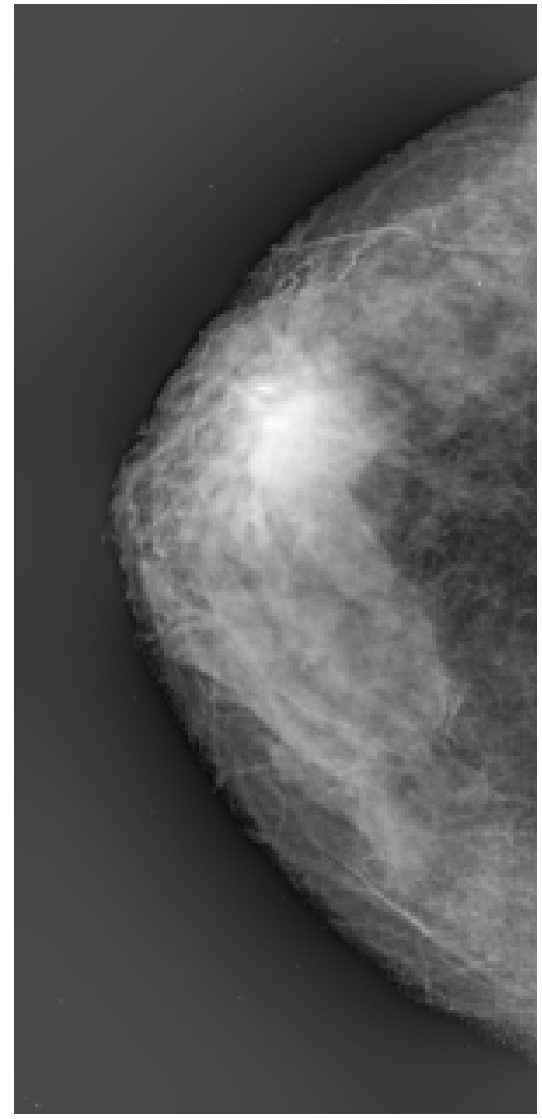

(c)

Figure 3: (a) Original mammogram. (b) Contrast enhancement by multiscale analysis [10]. (c) Enhancement obtained by fusion of enhanced features. 